\title{
Laser-frequency locking techniques for high-sensitivity strain measurements by high-birefringence fiber Bragg gratings and resonators
}

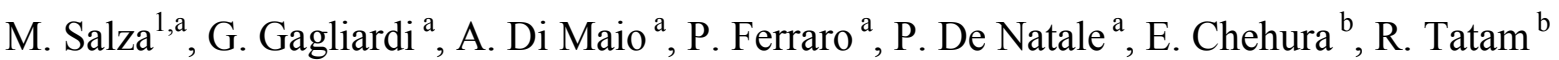 \\ ${ }^{a}$ Istituto Nazionale di Ottica Applicata INOA-CNR, Via Campi Flegrei, 34 I-80078 Napoli \\ ${ }^{\mathrm{b}}$ Centre for Photonics and Optical Engineering, School of Engineering, Cranfield University, \\ Bedford MK43 OAL, UK
}

\begin{abstract}
A new approach to simultaneously interrogate orthogonal axes of single Fiber-Bragg-Gratings (FBGs) and FBG-Fabry Perot resonator sensors fabricated in linearly highly birefringent (HiBi) fibre is presented. Novel interrogation techniques of single Fiber-Bragg-Gratings (FBGs) and FBG-resonator sensors are presented. For a single FBG, we combined a laser-modulation technique to an electronic feedback loop that keeps the source always frequency locked to one peak of the sensor's reflected spectrum. Two different lasers, with orthogonally-polarized states, were adopted to monitor simultaneously both the "fast" and "slow" FBG peaks. The corresponding correction signals from the servo-loop outputs can be interpreted as strain or temperature induced on the FBG. Detection limits ranging from $1 \mathrm{n} \varepsilon / \sqrt{ } \mathrm{Hz}$ to $100 \mathrm{n} \varepsilon / \sqrt{\mathrm{Hz}}$, for axial dynamic and static deformations, respectively, and of $0.025^{\circ} \mathrm{C} / \sqrt{\mathrm{Hz}}$ for temperature variations, are expected. A similar approach was developed for sub-pe resolution interrogation of an optical resonator made of a high-reflectivity FBG-pair, using the Pound-Drever-Hall (PDH) stabilization method..
\end{abstract}

Keywords: Fiber Bragg gratings, Pound-Drever-Hall method, laser-frequency modulation, high-birefringence fiber.

\section{INTRODUCTION}

In the area of optical fiber sensing, FBGs have been demonstrated to be the most promising mechanical probe for several research fields and applications. For this purpose, different interrogation systems have been developed so far, mostly based on broad-emission radiation sources combined to optical spectrum analyzers and filters. More recently, sophisticated schemes based on laser sources and frequency stabilization methods were devised, achieving unprecedented sensitivity levels for dynamic strain monitoring [1]. Among them, a significant contribution came also from the use of FBG-based resonant structures whose highly-dispersive power near resonance is exploited to measure sub-n $\varepsilon$ perturbations in a wide range of acoustic frequencies [2, 3]. However, in most such cases, low-acoustic frequency detection is affected by temperature cross-sensitivity effects that may limit the ultimate measurement accuracy. That is particularly relevant for field applications where a robust and reliable operation is required. A number of solutions have been proposed to avoid such limitation, including reference sensors, two-wavelengths FBGs, chirped gratings and birefringent fibers but often with serious degradation of the measurement precision [4]. In this work, we combine the advantages of recently-developed techniques that improve strain detection sensitivity, by means of laser-spectroscopic methods, to the use of FBG structures fabricated in high-birefringence (Panda) fibers. We describe the proposed methods, the experimental set-ups and the preliminary results obtained by a single FBG sensor and an in-fiber FabryPérot (FFP).

\section{METHODOLOGY}

A scheme of the interrogation set-up used for a Panda FBG is shown in figure 1. A distributed feedback (DFB) diode laser (1) and an extended-cavity diode laser (2), both equipped with single-mode Panda-fiber pig-tails, are used. The lasers are driven by highly-stable current and temperature controls, and their linear states of polarization (SOP) are aligned to the fiber slow and fast axes, respectively. Laser 1 is frequency modulated at $f_{l}=2 \mathrm{GHz}$ while laser 2 is modulated at $f_{2}=1 \mathrm{GHz}$ and both are coupled to the FBG through a PM 3-dB splitter. RF frequency modulation

${ }^{1}$ mario.salza@inoa.it; phone +39-0818675429; fax +39-0818675420; www.inoa.it/napoli

Third European Workshop on Optical Fibre Sensors, Antonello Cutolo, Brian Culshaw, José Miguel López-Higuera, Eds., Proceedings of SPIE Vol. 6619, 661915, (2007) · 0277-786X/07/\$18 · doi: 10.1117/12.738379 
generates two sideband frequencies at a relative distance from the carriers exactly equal to $f_{l, 2}$. Since the HiBi-FBG reflected signal presents two distinct peaks for slow and fast SOPs, they are first separated by a polarization splitter and then detected by two different photodiodes. A double-balanced mixer (DBM) demodulates the detector signals at frequency $f_{1,2}$ and extracts a dispersive-like signal resulting from sideband-carrier frequency beating. With this in mind, if the laser is perfectly resonant with the FBG, the mixer provides a voltage signal crossing zero in correspondence of the maximum reflectivity. Laser 1 and 2 give rise to mixer signals centered at slightly different wavelengths as a consequence of the fiber polarization-mode dispersion $(\sim 0.5 \mathrm{~nm})$. An example can be observed in figure 2 . In our technique, these signals are used as frequency discriminator (error) to stabilize the frequency of the lasers on the two Bragg peaks. This is accomplished by home-made proportional-integrative electronic loops (servo), which control laser current and external cavity using error signals as a reference. By active locking of each laser, the correction signals serve as strain monitors.

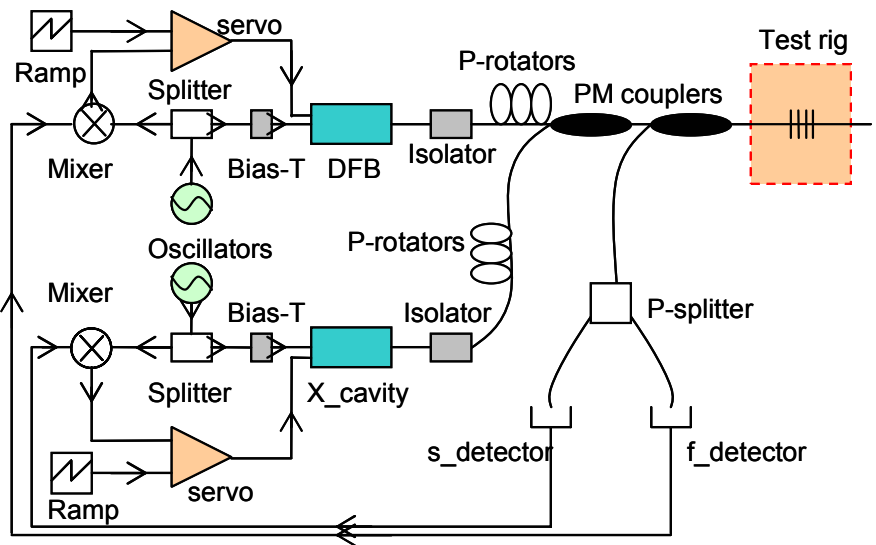

Fig. 1. Experimental set-up: P-rotators: polarization rotators; P-splitter: polarization splitter; X_cavity laser: extended cavity laser.

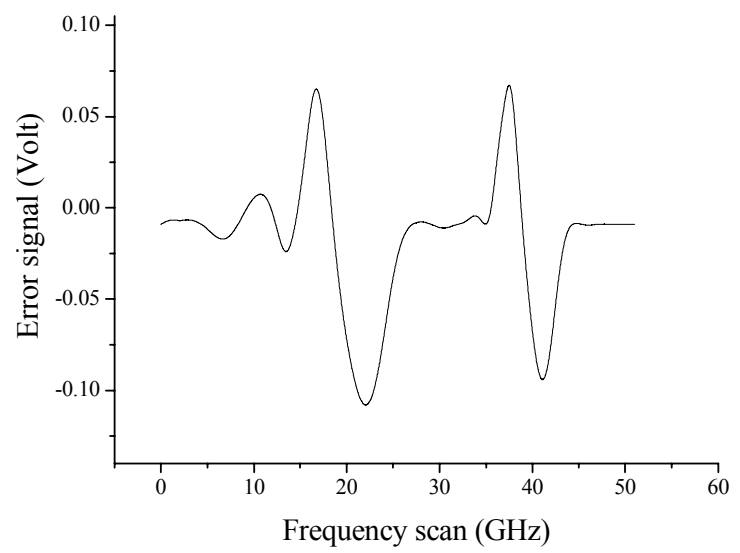

Fig. 2. Mixer output lineshapes around the PM FBG peaks for a $45^{\circ}$ incident polarization.

In our set-up, we could apply both longitudinal strain $\left(\varepsilon_{\mathrm{L}}\right)$ to the grating by a piezo-electric transducer (PZT) that stretched the fiber and transverse strain $\left(\varepsilon_{\mathrm{T}}\right)$ by pressing on the FBG length along the slow fiber axis by a test machine. The latter allowed to exert an evenly-distributed pressure between two glass surfaces in contact with the fiber. The actual mechanical deformations in all directions could be readily calibrated using a reference single-mode FBG, whose gauge factor was preliminary measured by applying known weights. Temperature variations $(\Delta \mathrm{T})$ could also be imposed to the fiber by means of a Peltier element in thermal contact with the FBG, the temperature being controlled by a PID stabilizer (resolution $\approx 0.01{ }^{\circ} \mathrm{C}$ ) and measured by an AD590 probe. Once the lasers were locked to the reflection peaks, for a given thermal or mechanical perturbation, we analyzed the corresponding changes in the servo feedback signal and retrieved the response of the PM FBG. By comparing all calibrations to the correction signal voltage, we obtained the final response factors of the system to the measurands, i.e. $\varepsilon_{\mathrm{L}}, \varepsilon_{\mathrm{T}}$ and $\Delta \mathrm{T}$, yielded by the two crossed-polarized lasers. Our method points out the possibility to interrogate simultaneously orthogonal axes of HiBi FBG sensors which can be of significance to applications in the measurement of multiple physical parameters. A similar but slightly more complicated procedure was employed for interrogation of the FFP. The instrumental arrangement is analogous to figure 1 while the FBG sensor is replaced by an in-fiber FBG resonator. Two high-reflectivity, Panda FBGs are coupled to form a FabryPérot structure. The Bragg mirrors are separated by about $50 \mathrm{~cm}$ and closed in aluminum cylinders to keep them mechanically insensitive. Here the sensitive element is represented by the intra-cavity fiber and thus small strain signals are excited by a PZT placed between the cylinders. Since the cavity has a nominal finesse exceeding 1000, even tiny effects affecting the fiber are strongly amplified, so that its intrinsic strain sensitivity is significantly increased [3]. In this case, the fiber birefringence plays a different role: each transmission resonance corresponding to a cavity mode is actually twofold if the incident SOP is not perfectly oriented along one of the principal axes. Static and dynamic deformations as well as thermal effects on the fiber results in changes of the optical pathlength and thus frequency shifts of the resonances, with different responses along the principal axes. As in the scheme described above, the lasers are locked to crossed-polarized peaks using the so-called Pound-Drever-Hall method [5]. According to considerations made above, each cavity resonance is split in a "slow" and a "fast" mode because the natural degeneracy of Fabry-Pérot resonator is removed by the fiber anisotropy. Hence, the signals that drive the locking circuits are very similar to the case shown in figure 2. As for laser locking, the electronics remained substantially the same but the modulation frequencies 
were chosen to be much lower, namely about $10 \mathrm{MHz}$. This is a significant simplification, since for our purpose it is sufficient that the sidebands are separated by a cavity linewidth to obtain a suitable error signal. Strain-induced shifts of the "slow" and "fast" resonances are monitored by the corrections generated by the servo loops and sent to the lasers. The calibration procedure can be implemented in the same manner as for a single sensor, provided a reference FBG is used for strain-to-voltage conversion.

\section{RESULTS}

Measurements with the single sensor have been carried out so far applying longitudinal loads by the PZT. In figure 3, linear fits to the graphs give longitudinal strain sensitivities of $1.264 \pm 0.02 \mathrm{pm} / \mu \varepsilon$ and $1.282 \pm 0.009 \mathrm{pm} / \mu \varepsilon$ for the slow and fast axes, respectively. Temperature sensitivities obtained for the same FBG were $10.326 \pm 0.02 \mathrm{pm} /{ }^{\circ} \mathrm{C}$ and $11.858 \pm 0.2 \mathrm{pm} /{ }^{\circ} \mathrm{C}$ for the slow and fast axes respectively (Figure 4). The results for longitudinal strain sensitivity of the HiBi FBG are barely distinguishable while temperatures are significantly different. Noise effects that limit measurement accuracy, e.g. spurious optical reflections, can be significantly reduced by using optical components with better anti-reflection coatings. Application experiments for this technique to the measurement of strain (longitudinal and transverse) and temperature, in both dynamic and static modes, have been performed and the results are currently being analysed and will be presented elsewhere.

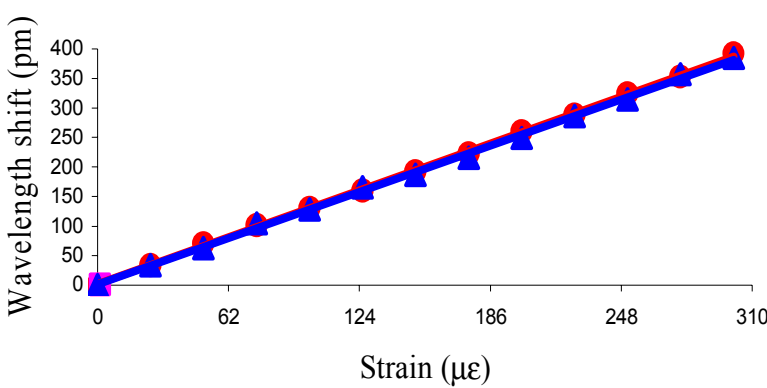

Fig. 3: Longitudinal strain response for PM FBG in both the fast and slow axes of the fiber $(1.264 \pm 0.02 \mathrm{pm} / \mu \varepsilon$ and $1.282 \pm$ $0.009 \mathrm{pm} / \mu \varepsilon$ for slow and fast axes respectively). $\bullet$ fast and $\boldsymbol{\Delta}$ slow axes.

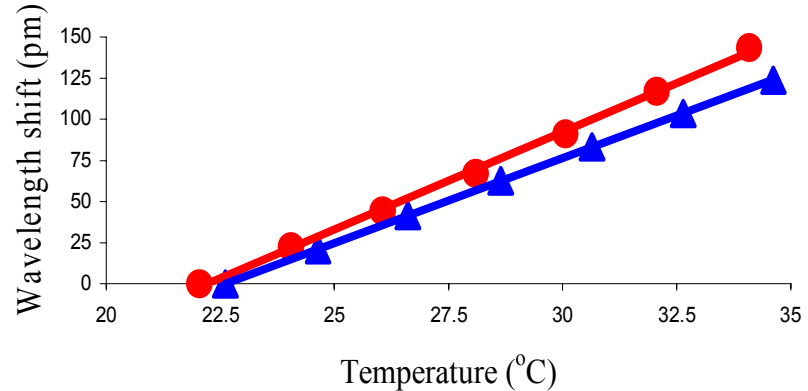

Fig. 4: Temperature response for PM FBG in both the slow and fast axes $\left(10.326 \pm 0.02 \mathrm{pm} /{ }^{\circ} \mathrm{C}\right.$ and $11.858 \pm 0.2 \mathrm{pm} /{ }^{\circ} \mathrm{C}$ for slow and fast axes respectively). $\bullet$ fast and $\boldsymbol{\Delta}$ slow axes.

As a proof of the efficiency of the sensing method, simultaneous detection of AC strain and temperature changes is demonstrated in figure 5. It is worth noting, from figure 5a, how the system discriminates between slow longitudinal strain and temperature variations applied at different frequencies by the slow-axis (laser 1) and the fast-axis (laser 2) signals. In figure $5 \mathrm{~b}$, the corresponding FFT traces are illustrated, exhibiting the peaks corresponding to mechanical ( 8 $\mathrm{Hz})$ and thermal $(0.5 \mathrm{~Hz})$ excitations in the fiber. A preliminary estimate suggests that quasi-static strain near to the 10 $\mathrm{n} \varepsilon$ level are within reach of our system, as can be deduced from figure $5 \mathrm{~b}$. At acoustic frequencies, we expect a gain of sensitivity of nearly a factor 100 . On the other hand, for small temperature changes, a noise-limited sensitivity of 0.008 ${ }^{\circ} \mathrm{C}$ in a $100 \mathrm{mHz}$ detection bandwidth can be extrapolated.
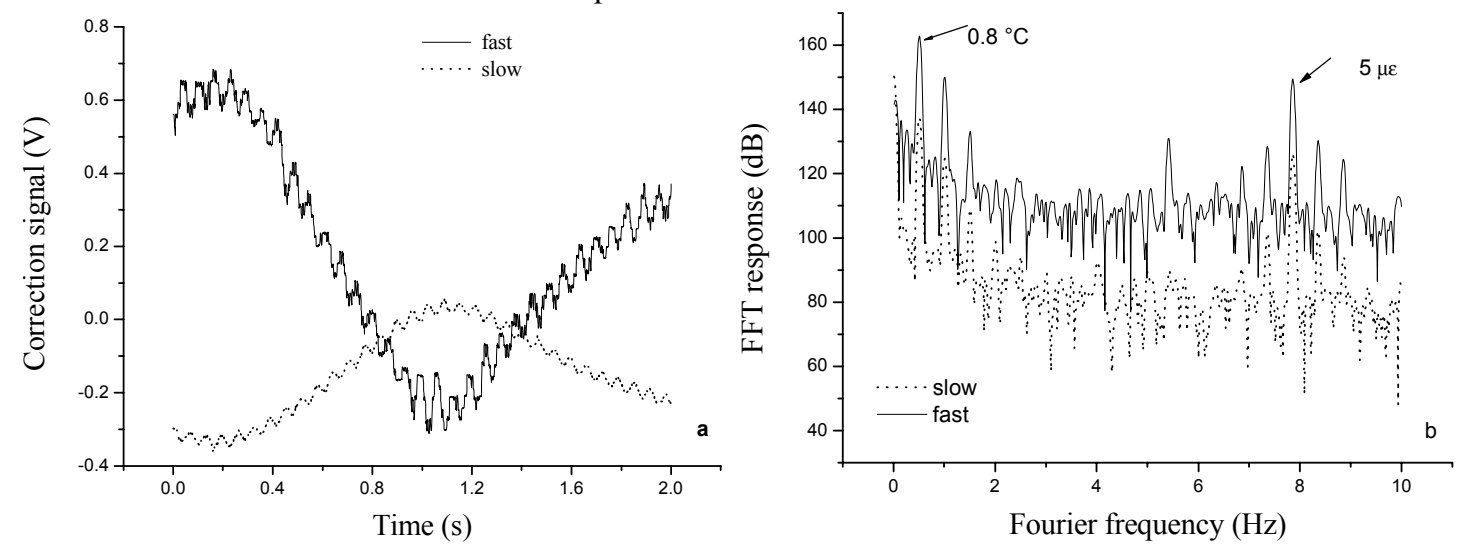

Fig. 5. a) Time response of the laser-locked system when a sine voltage is applied to the PZT $(8 \mathrm{~Hz})$ and a periodic temperature change is caused via the PID controller $(0.5 \mathrm{~Hz})$. b) FFT spectrum of the system response. 
The test experiment on the PM FFP has been carried out only for mechanical deformations so far. Using a low-voltage PZT, we applied static and dynamic (acoustic) strain along the intra-cavity fiber, while the two lasers are frequency locked to a couple of distinct, crossed-polarized resonances. As is visible in figure 6, from the sharp peak at $900 \mathrm{~Hz}$, the system is capable of detecting even smaller signals thanks to the resonator enhancement, showing a strain-noise level approaching $60 \mathrm{p} \varepsilon / \sqrt{ } \mathrm{Hz}$. Slight sensitivity degradation (by about a factor 10) is expected at lower frequency, mostly due to ambient noise and laser jitter contributions. Transverse strain as well as thermal stress effects on the PM sensor are still under study.

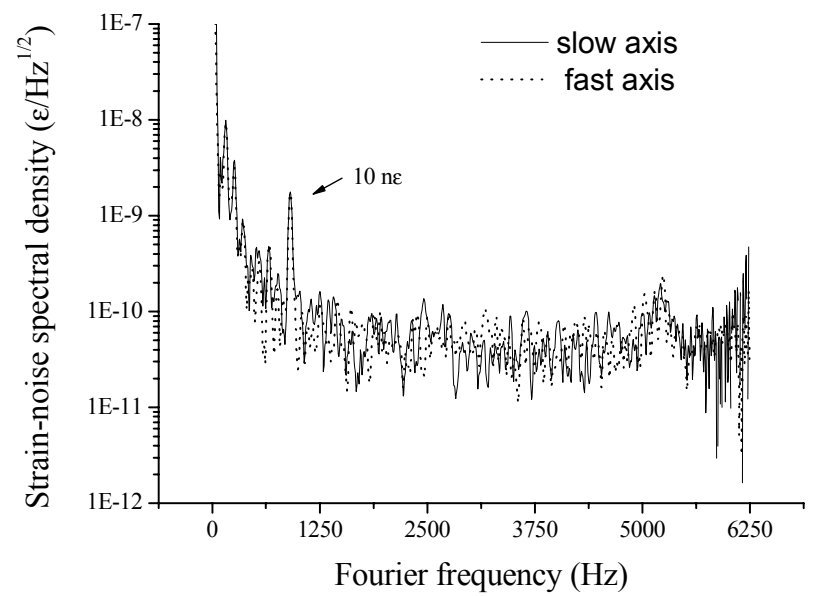

Fig. 6. Response to dynamic strain for fast and slow cavity modes in the laser-locked condition for a 10-ne signal on the intracavity fiber (resolution bandwidth $25 \mathrm{~Hz}$ ).

\section{CONCLUSIONS}

In this work, we present recent results on a novel interrogation scheme, relying on a laser-frequency stabilization technique, which enables for active tracking of FBG-based sensors fabricated in HiBi fiber. Both, a single Panda-fiber Bragg grating sensor and an intrinsically highly-reflectivity-FBG resonator were used in demonstrating the capability to perform simultaneous interrogation of the two orthogonal polarisation axes of a HiBi FBG thereby opening up prospects for strain-temperature discrimination, given high measured resolution possible with laser-based spectroscopic methods. Based on a preliminary estimate, minimum detectable strain signals below $10 \mathrm{n} \varepsilon / \sqrt{ } \mathrm{Hz}$ and $60 \mathrm{p} \varepsilon / \sqrt{ } \mathrm{Hz}$ can be in principle achieved for the single sensor and the fiber resonator, respectively. Quasi-static temperature variations of the order of few $\mathrm{mK}$ could be detected simultaneously by a single sensor. Interrogation and test of the fiber resonator in a similar fashion for simultaneous high-sensitivity strain and temperature measurements are in progress. Furthermore, an experimental investigation of cross-sensitivity effects of transverse strain and longitudinal strain in a dynamic measurement regime is in progress.

\section{REFERENCES}

1. B. Lissak, A. Arie, and M. Tur, "Highly sensitive dynamic strain measurements by locking lasers to fiber Bragg gratings," Opt. Lett. 23, 1930-1932 (1998).

2. J.H. Chow, D.E. McClelland, M.B. Gray, I.C. Littler, "Demonstration of a passive subpicostrain fiber strain sensor," Opt. Lett. 30, 1923-1925 (2005).

3. G. Gagliardi, M. Salza, P. Ferraro, P. De Natale, "Interrogation of FBG-based strain sensors by means of laser radio-frequency modulation techniques," J. Opt. A 8, S507-S513 (2006).

4. W. Urbanczyk, E. Chmielewska, and W.J. Bock, "Measurements of temperature and strain sensitivities of a two-mode Bragg grating imprinted in a bow-tie fibre," Meas. Sci. Technol. 12, 800-804 (2001)

5. R.W.P. Drever, J.L. Hall, F.V. Kowalski, J. Hough, G.M. Ford, A.J. Munley, and H. Ward, "Laser Phase and Frequency Stabilization Using an Optical Resonator,” Appl. Phys. B 31, 97-105 (1983). 